\title{
Treatment strategy for type 2 diabetes from the perspective of systemic vascular protection and insulin resistance
}

This article was published in the following Dove Press journal:

Vascular Health and Risk Management

3 July 2012

Number of times this article has been viewed

\section{Kazunori Utsunomiya}

Division of Diabetes, Metabolism and Endocrinology, Jikei University School of Medicine, Tokyo, Japan
Correspondence: Kazunori Utsunomiya Division of Diabetes, Metabolism and Endocrinology, Jikei University School of Medicine, Tokyo, Japan

$\mathrm{Tel}+8$ I 33433 IIII

Email kazu-utsunomiya@jikei.ac.jp
Abstract: This paper provides an update on the mechanisms of vascular impairment associated with insulin resistance and the pathogenesis of diabetic nephropathy and peripheral artery disease (PAD). It also considers the optimal treatment strategies for systemic vascular protection in light of recent findings. This area is of major clinical importance given the ongoing global epidemic of type 2 diabetes and the pivotal role played by insulin resistance in the mechanism of vascular impairment that manifests as macroangiopathy and microangiopathy. Timely diagnosis and intervention is critical in patients with systemic arteriosclerotic disease. Therefore, treatment strategies are aimed not only at targeting the presenting pathology, but also at reducing the risk of cardiovascular events. These efforts can help reduce the risk of both cardiovascular events and mortality. Treatment for PAD includes pharmacotherapy, endovascular treatment, and vascular reconstruction, along with exercise therapy. Because PAD can cause ischemia in the lower extremities, typical drug approaches include use of vasodilators and antiplatelet agents. Beraprost sodium and cilostazol are common choices in Japan, and their risks and benefits are discussed. Of note, beraprost has several therapeutic properties, including vascular endothelial protection, and antiplatelet and anti-inflammatory effects, in addition to vasodilatory activity. In patients with PAD, these activities improve the pathological process in the lower extremities and reduce the incidence of systemic vascular events. Recent preclinical findings indicate that beraprost improves not only ischemic extremities through its vasodilatory properties, but also reduces the insulin resistance which affects vascular endothelium. In this way, beraprost may contribute to an overall systemic vascular protective action. The use of agents, such as beraprost, which are capable of improving insulin resistance and resulting vascular endothelial function at an earlier disease stage, may ultimately contribute to increasing the life expectancy of patients with PAD.

Keywords: peripheral artery disease, insulin resistance, beraprost, vascular, protection

\section{Introduction}

In recent years, close links have gradually been revealed between insulin resistance and the pathogenesis of a number of diseases. Vascular complications of diabetes can be largely classified into macroangiopathy, eg, cardiovascular disease, stroke, and peripheral artery disease (PAD), and microangiopathy, eg, diabetic nephropathy. These two classes of diseases have traditionally been regarded as the result of different pathological mechanisms. However, the findings of recent research efforts show that these diseases share insulin resistance as the common primary pathological pathway. The purpose of this review is to update physicians about recent progress on the mechanisms of vascular impairment associated with insulin resistance and 
the pathology of diabetic nephropathy and PAD, and to discuss current therapeutic strategies for systemic vascular protection.

\section{Paradigm shifts}

Diabetic vascular complications consist of microangiopathy causing abnormalities at the capillary level, and macroangiopathy which is primarily the result of arteriosclerosis. Macroangiopathy is associated with pathophysiological conditions, including insulin resistance and metabolic syndrome, ${ }^{1-3}$ whereas microangiopathy, including diabetic nephropathy, is considered to be vascular failure secondary to hyperglycemia. As such, the pathophysiological mechanisms of these two types of vascular disease have been traditionally recognized as different. This is true in the case of type 1 diabetes, where only a hyperglycemic state contributes to the development of diabetic nephropathy. However, recent studies have clearly demonstrated that accumulation of clinical traits in the metabolic syndrome (MetS) (Figure 1) could be a risk factor for development of chronic kidney disease (CKD), suggesting a contribution of insulin resistance to progression of CKD. ${ }^{4}$ It was also recently been reported that mice lacking insulin receptors in glomerular podocytes show glomerular sclerosis. ${ }^{5}$ These findings suggest that insulin resistance may play a crucial and common pathophysiological role not only in cardiovascular disease, ie, macroangiopathy, but also in CKD, ie, microangiopathy.

Diabetes is a major cause of CKD, but the risk for CKD may already be elevated during the prediabetes phase associated with insulin resistance, as is known to be the case with cardiovascular disease (Figure 1). In contrast with type 1 diabetes, vascular complications arise from highly complicated pathophysiology in type 2 diabetes where vascular damage due to an insulin-resistant state can be worsened by diabetes-induced hyperglycemia.

\section{PAD as a cardiovascular risk factor}

PAD is a macroangiopathy that can develop as arteriosclerotic progresses, suggesting a strong causal relation with insulin resistance. This is supported by study findings showing that the higher the HOMA-IR (homeostatic model assessment of insulin resistance) value becomes, the more the prevalence of PAD increases to a clinically significant extent (Figure 2). ${ }^{6}$ PAD is a peripheral vascular disease involving ischemia to the lower extremities followed by intermittent claudication and ultimately necrosis during progression of the disease. This typically requires amputation of the affected extremity, which invariably compromises patient quality of life to a devastating extent. This macroangiopathy is characterized not only by substantial deterioration in quality of life secondary to lower extremity ischemia, but, also importantly, by a poor prognosis and a worse long-term outcome. ${ }^{7}$

During the time course of development of PAD, along with symptomatic PAD, less intensive, asymptomatic conditions are also associated with a high incidence of cardiovascular events (Figure 3 ). ${ }^{8}$ This underscores the necessity of timely diagnosis and earlier treatment intervention because these efforts can greatly determine the extent of any reduction in risk of both cardiovascular events and mortality. These fac-

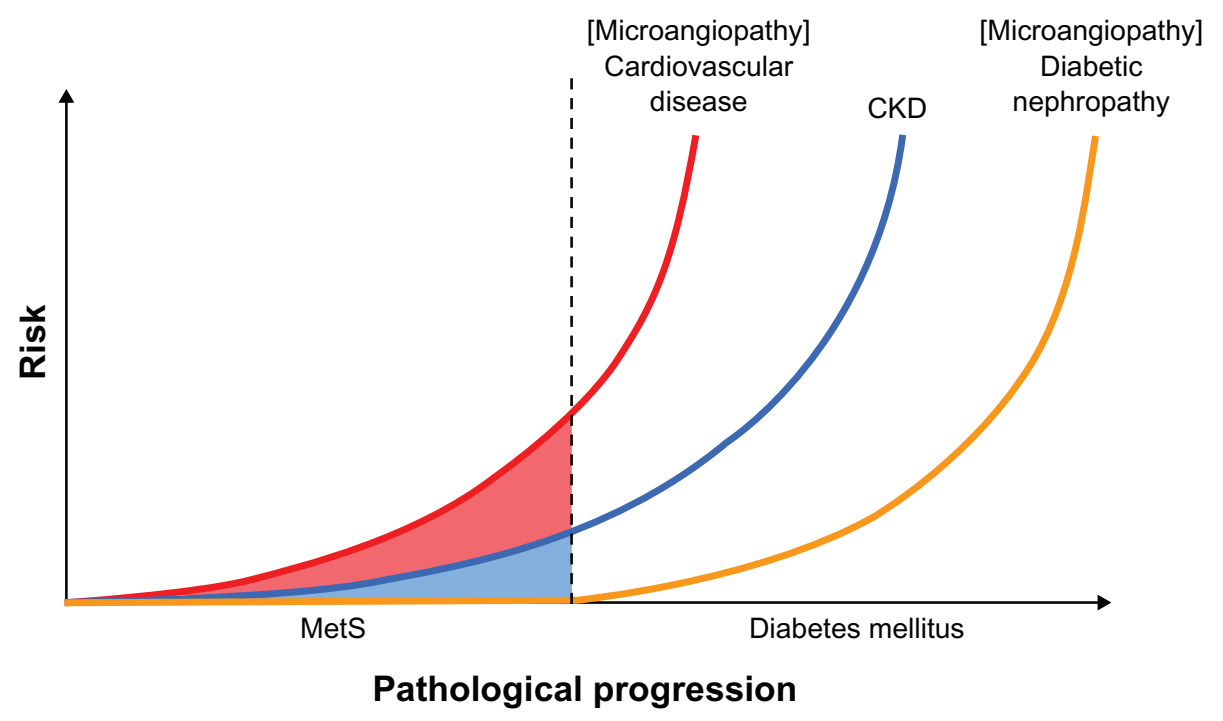

Figure I Risk of disease aggravation with diabetes progression.

Abbreviations: CKD, chronic kidney disease; MetS, metabolic syndrome. 


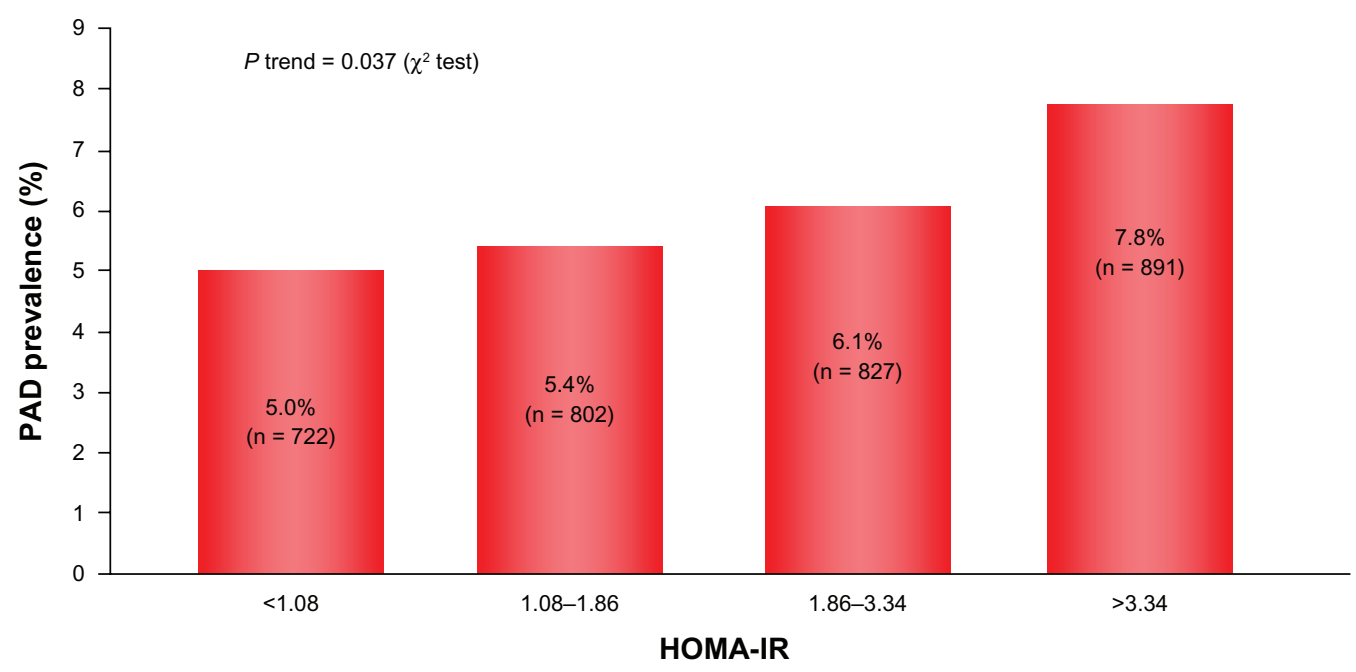

Figure 2 Relationship between insulin resistance and PAD.

Reena L, et al. Circulation. 2008; I 18:33-4I. (C) 2008 Wolters Kluwer Health.

Abbreviations: HOMA-IR, homeostatic model assessment of insulin resistance; PAD, peripheral artery disease.

tors indicate that insulin-resistant patients should be checked for the possibility of PAD, followed by correct diagnosis and appropriate treatment.

In addition to the earlier discussion on the potential relationship between CKD and insulin resistance, a correlation between PAD and CKD has also, and perhaps more intriguingly, been established. Data from the US National Health and Nutrition Examination Survey conducted from 1999 to 2002 documented that a reduction of estimated glomerular (eGFR) (Figure 4) filtration rate, especially below $60 \mathrm{~mL} /$ $\min / 1.73 \mathrm{~m}^{2}$ (the diagnostic threshold for CKD stage 3), markedly increased the risk of developing PAD (Figure 4). ${ }^{9}$
Furthermore, a separate study into the possible relationship between CKD and PAD in Asian patients revealed that the prevalence of PAD increases with CKD progression stage. Patients with concurrent CKD and PAD demonstrated elevated cardiovascular mortality and an overall mortality higher compared with patients with either PAD alone or CKD alone, indicating that the complication of CKD in the presence of PAD may strongly increase the risk of cardiovascular disease (Figure 5). ${ }^{10}$

Overall, these findings illustrate a strong association between PAD and CKD, both of which share the common underlying pathophysiology of insulin resistance leading to

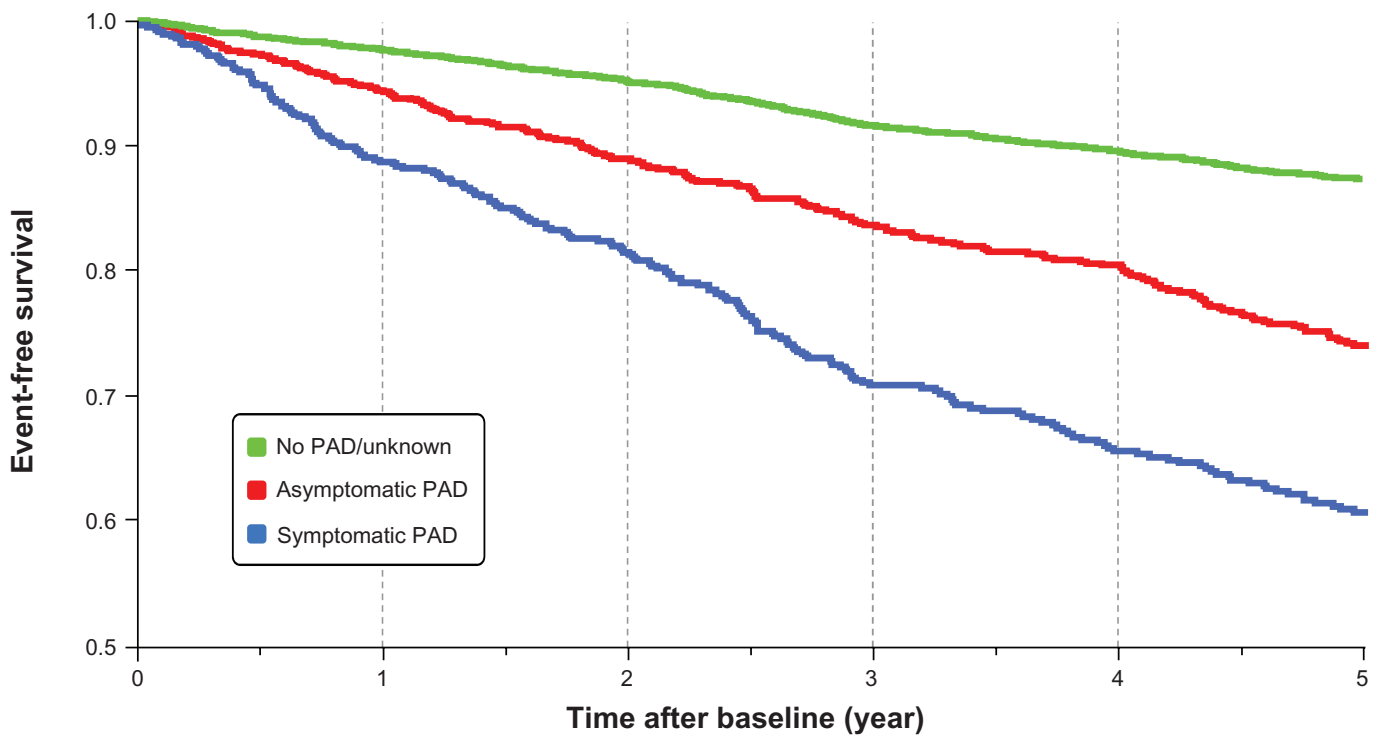

Figure 3 Life expectancy in PAD patients.

Diehm, et al. Circulation. 2009;120:2053-206I. (c) 2008 Wolters Kluwer Health.

Abbreviation: PAD, peripheral artery disease. 


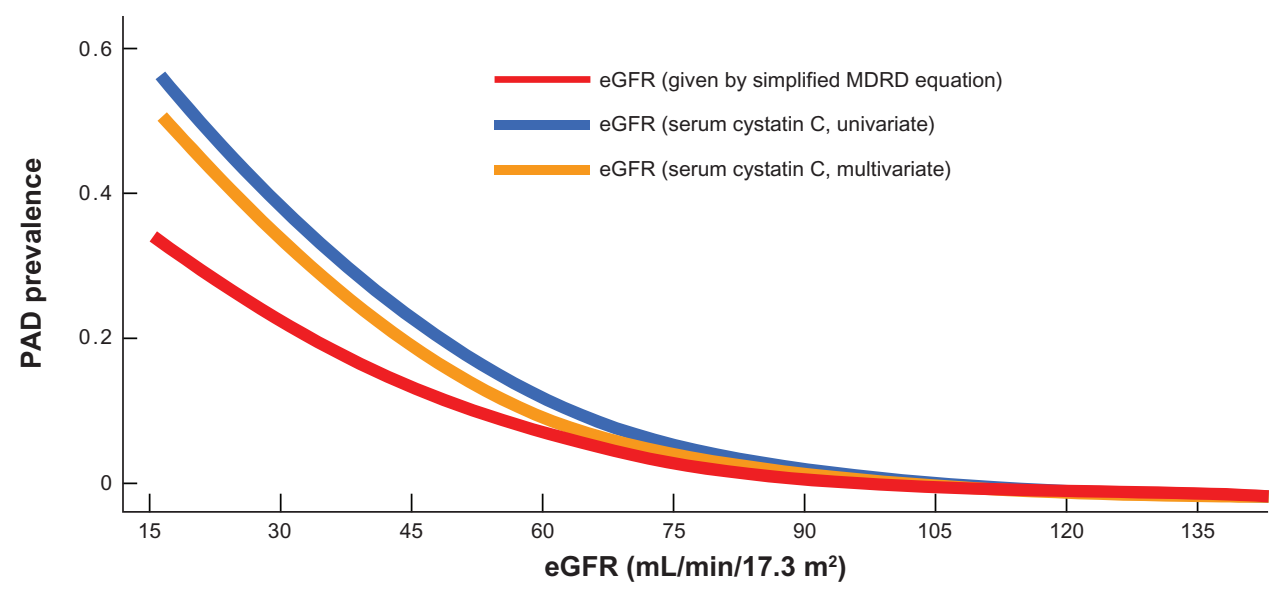

Figure 4 Relationship between eGFR levels and PAD prevalence.

Selvin E, et al. Eur Heart J. 2009;30:1918-1925. (C) 2010 SAGE Publications.

Abbreviation: PAD, peripheral artery disease; eGFR, estimated glomerular filtration rate.

vascular impairment. This fact warrants not only a therapeutic approach targeting PAD, but also effective and timely management of cardiovascular risk in patients with systemic arteriosclerotic disease.

\section{Drugs for PAD and approaches to vascular protection}

Current treatment for PAD includes drugs, endovascular treatment, and vascular reconstruction, in addition to exercise therapy. Because PAD causes ischemia in the lower extremities secondary to arteriosclerotic stenosis and occlusion, typical drug approaches include vasodilators and antiplatelet agents. In Japan, frequent choices are beraprost and cilostazol, amongst others. ${ }^{11}$ Table 1 provides a list of oral agents currently used in the clinical setting in Japan. Cilostazol is a phosphodiesterase 3 inhibitor, and is recommended by the TASC-II PAD treatment guideline for improvement of intermittent claudication in patients with PAD. ${ }^{12}$

Treatment with a phosphodiesterase 3 inhibitor is also indicated to prevent recurrence in the post-cerebral infarction stage, and is positioned as a compound effective for vascular protection in the brain and lower extremities. However, of note, phosphodiesterase 3 also exists abundantly in myocardial cells and, therefore, requires caution with regard to possible adverse events, such as arrhythmia. It should be noted that beraprost is derived from an endogenous substance, prostaglandin $\mathrm{I}_{2}$, and has several therapeutic properties, including vascular endothelial protection and antiplatelet and anti-inflammatory effects, in addition to vasodilatory activity. ${ }^{13}$ In PAD, the clinical benefits of beraprost include improving impaired circulation in the lower extremities. ${ }^{14}$ The role of beraprost is in reduction of the incidence of systemic vascular events, as demonstrated by meta-analysis of

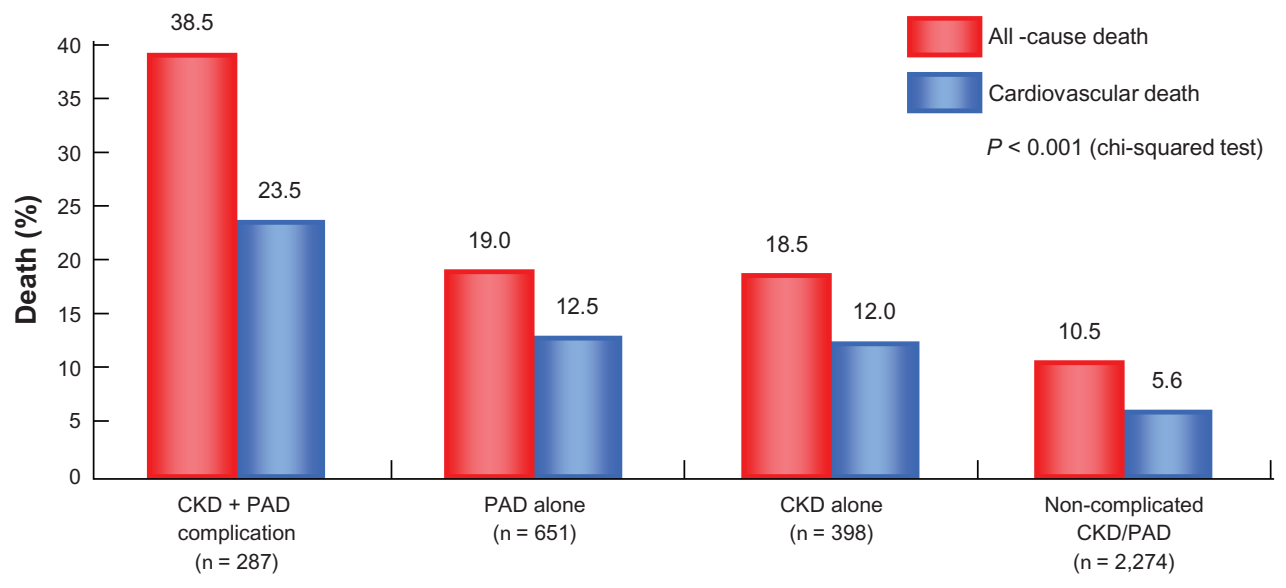

Figure 5 Relationship between CKD/PAD complication and cardiovascular death. Luo Y, et al. Vasc Med. 2010;15:107-II2. () 2010 SAGE Publications. Abbreviations: CKD, chronic kidney disease; PAD, peripheral artery disease. 


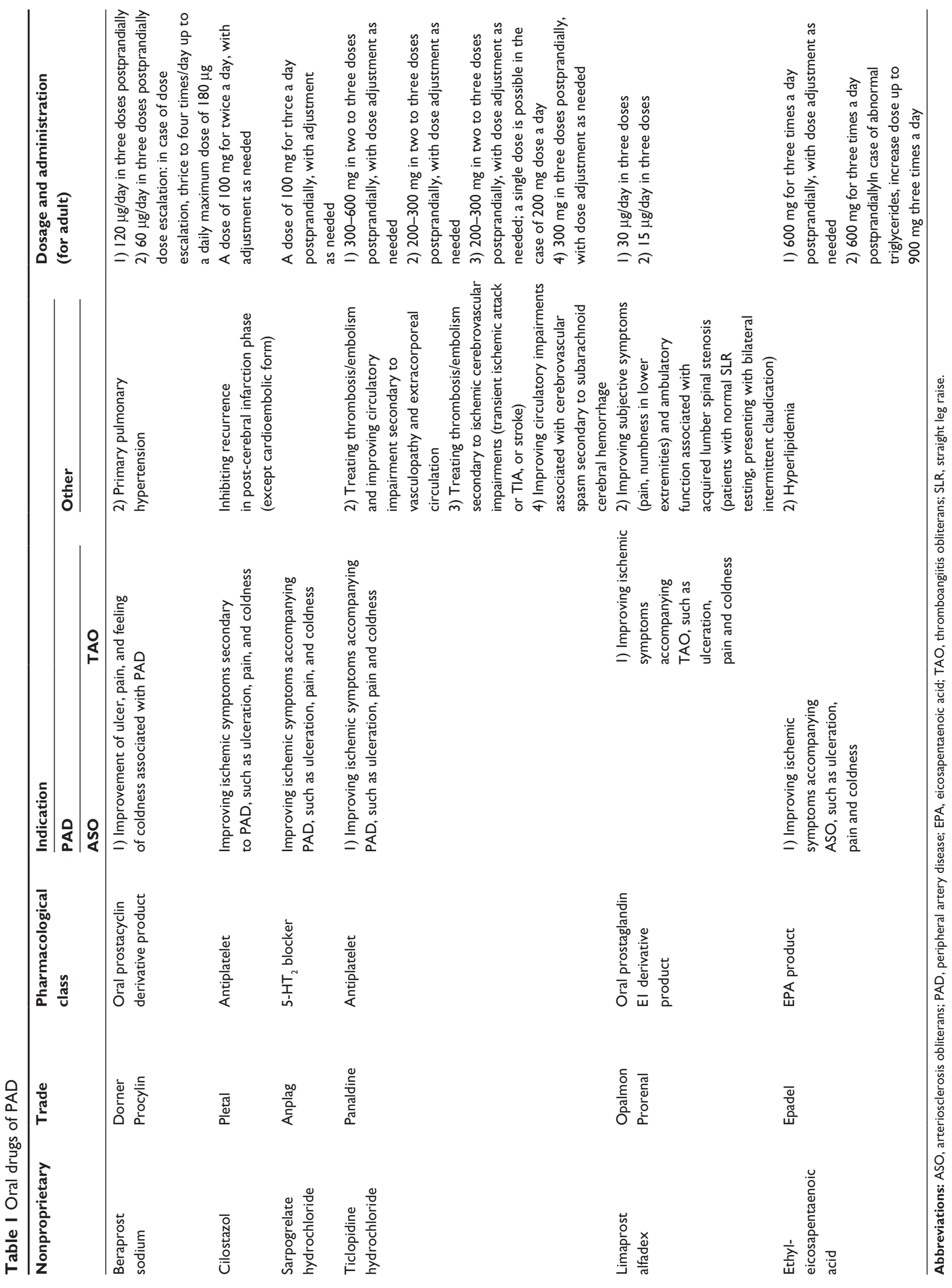


two double-blind, placebo-controlled studies. This analysis included 594 patients who received beraprost and 590 who received placebo. Primary endpoints were defined as vascular events, including lower limb deterioration and cardiovascular and cerebrovascular events. The statistical analysis was performed using the Mantel-Haenszel Chi-square test based on intent to treat. The results showed that the risk ratio was $0.608(P=0.012)$, demonstrating the beneficial effect of beraprost on all vascular events. ${ }^{15}$ This effect is attributed to the multimodal pharmacodynamic properties of beraprost related to its antiplatelet activity and vascular endothelial protection.

A recent animal study demonstrated that insulin signaling in vascular endothelium greatly contributes to insulin sensitivity in peripheral skeletal muscles (Figure 6), and therefore to pathogenesis of microangiopathy and macroangiopathy. ${ }^{16}$ Kubota et $\mathrm{al}^{16}$ reported that reduced function of vascular endothelium results in reduced insulin sensitivity; they demonstrated that impaired insulin signaling in endothelial cells, due to reduced insulin receptor substrate 2 expression and insulin-induced phosphorylation of endothelial nitric oxide synthase, caused attenuation of insulin-induced capillary recruitment and insulin delivery, which in turn reduced glucose uptake by skeletal muscle. Moreover, restoration of insulin-induced phosphorylation of endothelial nitric oxide synthase in endothelial cells completely reverses reduction in capillary recruitment and insulin delivery. Beraprost ameliorated the permeability of skeletal capillaries and improved insulin transfer into skeletal muscles. These studies also demonstrated that beraprost not only improves ischemic extremities through its vasodilatory properties, but also reduces insulin resistance which affects vascular endothelium, suggesting that beraprost may contribute to an overall systemic vascular protective action.

One of the traditional goals of treatment for PAD is to improve the classical symptoms, including intermittent claudication and a sensation of coldness. Recent research has indicated that PAD, even in the earlier asymptomatic stages, may increase the risk for cardiovascular diseases, underlying the importance of efforts not only to alleviate the symptoms of PAD, but also to reduce the risks for cardiovascular disease. In view of the strong association between microangiopathy, macroangiopathy, and insulin resistance-induced vascular impairment or inflammation as discussed earlier, the focus of comprehensive treatment for PAD-induced vascular abnormalities should target not only the site specifically affected, eg, the lower extremities, but also the common underlying pathophysiology, specifically to improve the vascular endothelium and inhibit inflammation. This inclusive therapeutic approach has the potential to achieve the desired systemic vascular protection. In summary, the use of drugs capable of improving insulin resistance and vascular endothelial function at an earlier stage of the disease may ultimately contribute to increasing the life expectancy of patients with PAD. Therefore, consideration of their wider use at earlier stages of PAD is warranted.

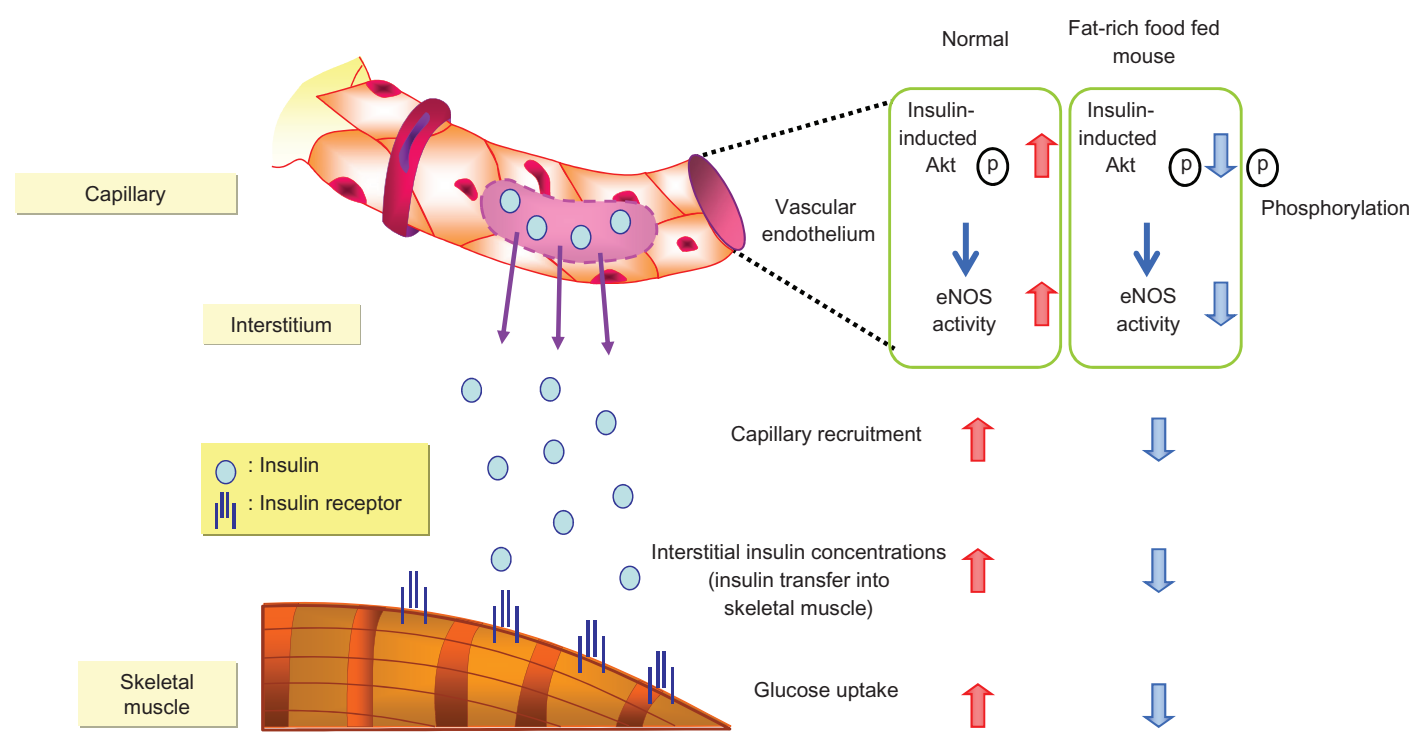

Figure 6 Insulin transfer from vascular endothelial cells to skeletal muscle. Kubota T, et al. Cell Metab. 20I I;13:294-307. (c) 20I I Elsevier. 


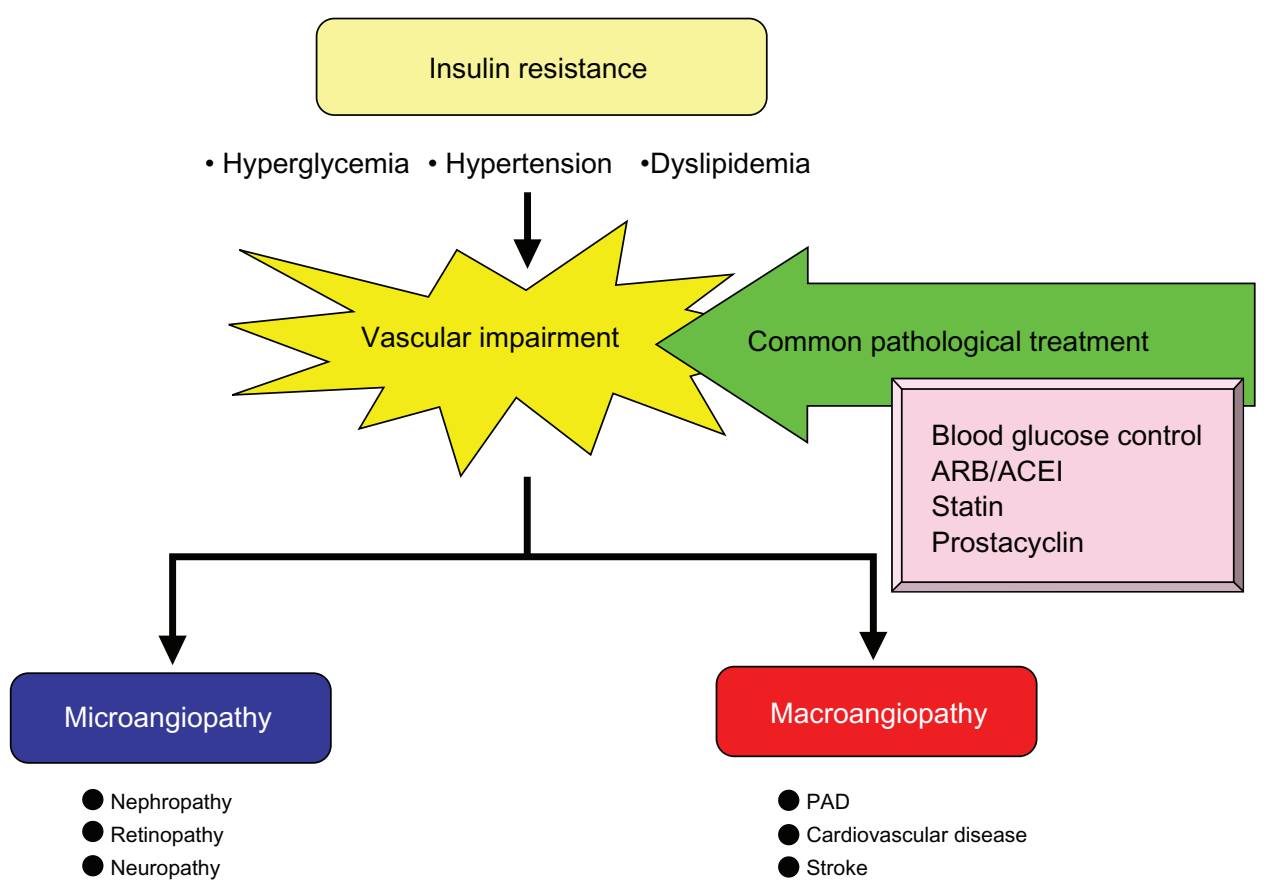

Figure 7 Development of vascular impairment in insulin-resistant state and treatment.

Abbreviations: PAD, peripheral artery disease; ARB, angiotensin receptor blocker; ACEI, angiotensin converting enzyme inhibitor.

\section{Insulin resistance-targeted treatment approach}

Insulin resistance is a serious pathophysiological condition which can result in impairment of the entire vasculature irrespective of vessel size, and thereby cause diabetesinduced vascular complications. In addition, recent research has revealed that CKD is closely associated with cardiovascular disease risk in the presence of PAD. As shown in Figure 7, these complications share a common underlying pathological mechanism in the form of insulin resistance. Indeed, disorders such as hypertension, insulin resistance, type 2 diabetes, obesity, and subsequent cardiovascular disease are intertwined at a pathological level and currently represent epidemics that pose a major public health challenge worldwide. Meeting this challenge requires an understanding of the interplay between adipose tissue and the vasculature. Macrovascular and microvascular dysfunction is important not only in the development of obesity-related target organ damage, but also in the development of cardiovascular risk factors, including hypertension and insulin resistance. ${ }^{17,18}$ It should be noted that in cardiovascular disease, type 2 diabetes and hypertension are characterized by resistance to insulin-mediated glucose disposal. Furthermore, insulin resistance and the compensatory hyperinsulinemia associated with insulin resistance are independent predictors of all three clinical syndromes. Therefore, it seems reasonable to suggest that insulin resistance is the link between overweight/ obesity and the adverse clinical syndromes related to excess adiposity. ${ }^{17,18}$

Therefore, it would seem logical to interrupt these negative associations at an earlier stage of the disease process using a treatment approach that takes into consideration the extra dimension of systemic vascular protection. The conventional therapeutic approach emphasizes, first of all, management of blood glucose levels, as well as blood pressure control by the use of angiotensin receptor blockers and angiotensin-converting enzyme inhibitors along with lipid management through the use of statin therapy. However, for vascular impairment induced by insulin resistance, prostacyclins may now be considered alongside the conventional options. It is anticipated that ongoing studies and emerging evidence will demonstrate yet further beneficial effects of the prostacyclins on reducing the incidence of vascular events at an earlier stage of the disease.

\section{Disclosure}

The authors report no conflicts of interest in this work.

\section{References}

1. Holman RR, Paul SK, Bethel MA, Matthews DR, Neil HA. 10-year follow-up of intensive glucose control. N Engl J Med. 2008;359:1577-1589.

2. Stamler, Vaccaro O, Neaton JD, Wentworth D. Diabetes, other risk factors, and 12-year cardiovascular mortality for men screened in the Multiple Risk Factor Intervention Trial. Diabetes Care. 1993;16:434-444. 
3. Hedbland B, Nilsson P, Engstrom G, Berglund G, Janzon L. Insulin resistance in non-diabetic subjects is associated with increased incidence of myocardial infarction and death. Diabet Med. 2002;19:470-475.

4. Kurella M, Lo JC, Chertow JM. Metabolic syndrome and the risk for chronic kidney disease among nondiabetic adults. J Am Soc Nephrol. 2005;16:2134-2140.

5. Welsh GI, Hale LJ, Eremina V, et al. Insulin signaling to the glomerular podocyte is critical for normal kidney function. Cell Metab. 2010;12:329-340.

6. Pande RL, Perlstein TS, Beckman JA, Creager MA. Association of insulin resistance and inflammation with peripheral arterial disease: the National Health and Nutrition Examination Survey, 1999 to 2004. Circulation. 2008;118:33-41.

7. Hisao Kumakura. Life prognosis of occlusive arteriosclerosis: relations among risk factors, complications and treatment methods. J Jap Coll Angiol. 2002;42:889-895.

8. Diehm C, Allenberg JR, Pittrow D, et al. Mortality and vascular morbidity in older adults with asymptomatic versus symptomatic peripheral artery disease. Circulation. 2009;120:2053-2061.

9. Selvin E, Kottgen A, Coresh J. Kidney function estimated from serum creatinine and cystatin $\mathrm{C}$ and peripheral arterial disease in NHANES 1999-2002. Eur Heart J. 2009;30:1918-1925.

10. Luo Y, Li X, Li J, et al. Peripheral arterial disease, chronic kidney disease, and mortality: the Chinese Ankle Brachial Index Cohort Study. Vasc Med. 2010;15:107-112.
11. Shigematsu H, Nishibe T. Three-year cardiovascular events and disease progress in patients with peripheral arterial disease: results from Japan Medication Therapy for Peripheral Arterial Disease (J-METHOD). Int Angiol. 2010;29(2 Suppl 1):2-13.

12. Norgren L, Hiatt WR, Dormandy JA, et al. Inter-Society Consensus for the Management of Peripheral Arterial Disease (TASC-II). Eur $J$ Vasc Endovasc Surg. 2007;33 Suppl 1:S1-S75.

13. Kawabe J, Ushikubi F, Hasebe N. Prostacyclin in vascular diseases: recent insights and future perspectives. Circ J. 2010;74:836-843.

14. Lièvre M, Morand S, Besse B, Fiessinger JN, Boissel BP. Oral beraprost sodium, a prostaglandin $\mathrm{I}_{2}$ analogue for intermittent claudication: a double-blind randomized, multicenter controlled trial. Circulation. 2000;102:426-431.

15. Origasa H, Ikeda Y, Shimada K, Shigematsu H. Oral beraprost sodium as a prostaglandin I2 analogue for vascular events in patients with peripheral arterial disease: meta-analysis of two placebo-controlled randomized trials. Jpn J Pharmacoepidemiol. 2004;9:45-51.

16. Kubota T, Kubota N, Kumagai H, et al. Impaired insulin signaling in endothelial cells reduces insulin-induced glucose uptake by skeletal muscle. Cell Metab. 2011;13:294-307.

17. De Boer MP, Meijer RI, Wijnstok NJ, et al. Microvascular dysfunction: a potential mechanism in the pathogenesis of obesity-associated insulin resistance and hypertension. Microcirculation. 2012;19:5-18.

18. Reaven GM. Insulin resistance: the link between obesity and cardiovascular disease. Med Clin North Am. 2011;95:875-892.
Vascular Health and Risk Management

\section{Publish your work in this journal}

Vascular Health and Risk Management is an international, peerreviewed journal of therapeutics and risk management, focusing on concise rapid reporting of clinical studies on the processes involved in the maintenance of vascular health; the monitoring, prevention and treatment of vascular disease and its sequelae; and the involvement of

\section{Dovepress}

metabolic disorders, particularly diabetes. This journal is indexed on PubMed Central and MedLine. The manuscript management system is completely online and includes a very quick and fair peer-review system, which is all easy to use. Visit http://www.dovepress.com/ testimonials.php to read real quotes from published authors. 